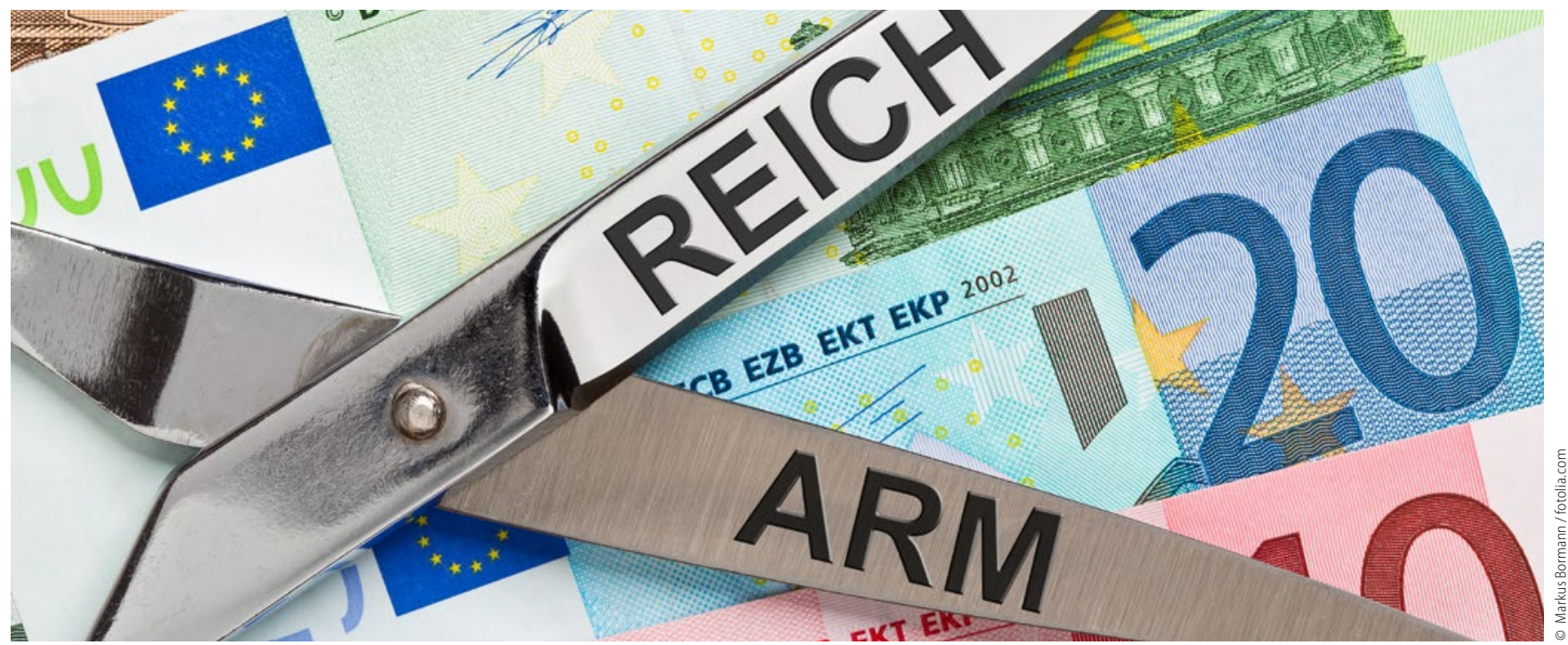

Bericht "Gesundheit in Deutschland" 2015

\title{
Auch bei den Zähnen zeigt sich der soziale Unterschied
}

\author{
Mehr als 500 Seiten stark und über 2,2 Kilogramm schwer: Der 3. Bericht "Gesundheit in \\ Deutschland" ist ein echtes Schwergewicht. In elf Kapiteln gibt er einen fundierten Überblick \\ über den Stand und die Entwicklung der Gesundheit der Menschen in Deutschland, welche \\ Krankheiten auf dem Vormarsch sind (vor allem Diabetes), die zehn häufigsten Todesursachen \\ und wie hoch die Lebenserwartung ist. In einem kleinen Teil des Berichts geht es auch um die \\ Zahngesundheit in Deutschland.
}

Der Bericht „Gesundheit in Deutschland“ ist Teil der Gesundheitsberichterstattung des Bundes, die am Robert-Koch-Institut angesiedelt ist. „Unser Ziel ist es, dass sich alle Menschen in Deutschland, unabhängig von Wohnort und Geldbeutel, auch zukünftig auf eine hochwertige medizinische Versorgung verlassen können“, sagte Bundesgesundheitsminister Hermann Gröhe (CDU) bei der Vorstellung des Berichts in Berlin. Das könnte sich auch als notwendig erweisen, denn laut Bericht sind Menschen mit niedrigem sozioökonomischem Status häufiger von Krankheiten, Beschwerden und Behinderungen betroffen als andere.

\section{Weniger Karies im Kindesalter}

Dies zeigt sich auch im Kapitel über die Mundgesundheit. Positiv für die Zahngesundheit in Deutschland: Die Karieshäufigkeit hat sich in fast allen Altersgruppen deutlich verringert, bei Erwachsenen und älteren Menschen ist erstmals ein Rückgang $\mathrm{zu}$ verzeichnen. Besonders deutlich ist demnach die Verbesserung der Zahngesundheit im Kindesalter. Als wesentliche Ursache für die insgesamt verringerte Karieshäufigkeit nennt der Bericht ein besseres Vorsorgeverhalten, das unter anderem mit regelmäßigem Zähneputzen und der Inanspruchnahme der zahnärztlichen Kontrolluntersuchungen einhergeht. Aber auch hier machen sich soziale Unterschiede bemerkbar: Demnach zeigt sich eine deutlich höhere Karieshäufigkeit bei Kindern aus Familien mit niedrigem Sozialstatus. Und noch etwas wird in dem Bericht deutlich: Es gibt Unterschiede bei den Altersgruppen. Während die Prävalenz bei Schulkindern deutlich rückläufig ist, stagniert sie bei Klein- und Vorschulkindern. Grund dafür ist nach Ansicht der Experten die nach wie vor starke Verbreitung der frühkindlichen Karies. Sieben bis 20 Prozent der Ein- bis Sechsjährigen haben eine so genannte SaugflaschenKaries. Ursache ist das Nuckeln zuckerhaltiger Getränke aus Nuckelflaschen. Auffällig ist auch hier der soziale Zusammenhang. Ein Großteil der Kinder mit Milchzahnkaries kommt aus sozial schlechter gestellten oder bildungsfernen Familien.

Insgesamt, so stellt der Bericht fest, hat sich die Zahngesundheit in Deutschland aufgrund erfolgreicher Prävention und guter zahnärztlicher Versorgung seit Ende der 1990er-Jahre aber deutlich verbessert. Um diesen Erfolg auch zukünftig zu sichern, seien besondere Anstrengungen im Bereich der Prävention der frühkindlichen Karies, von Milchzahnkaries sowie Parodontalerkrankungen notwendig. Dabei seien frühzeitige Diagnose sowie eine konsequente Nachsorge der Parodontitis auch in Hinblick auf den demografischen Wandel und der damit verbundenen immer älter werdenden Gesellschaft von großer Bedeutung.

Peter Königsfeld 